\title{
A NOTE ON CONVEXITY, CONCAVITY, AND GROWTH CONDITIONS IN DISCRETE FRACTIONAL CALCULUS WITH DELTA DIFFERENCE
}

\author{
CHRISTOPHER S. GOODRICH
}

\begin{abstract}
We demonstrate that some recent results regarding the connection between the convexity of the map $t \mapsto f(t)$ and the sign of $\Delta_{a}^{v} f(t)$, with $2<v<3$, can be improved. In particular, by utilizing a recent inequality due to Jia, Erbe, and Peterson, we are able to improve some of the existing results in the literature. As part of this study we illustrate the improvements that our results afford by providing several specific examples of their application.
\end{abstract}

Mathematics subject classification (2010): Primary: 26A51, 39A12, 39B62; Secondary: 26A33, 39A70, 39A99.

Keywords and phrases: Discrete fractional calculus, convexity, concavity, Taylor monomial, delta difference.

\section{REFERENCES}

[1] F. M. Atici, N. ACAR, Exponential functions of discrete fractional calculus, Appl. Anal. Discrete Math. 7 (2013), 343-353.

[2] F. M. Atici, P. W. Eloe, Initial value problems in discrete fractional calculus, Proc. Amer. Math. Soc. 137 (2009), 981-989.

[3] F. M. Atici, P. W. Eloe, Two-point boundary value problems for finite fractional difference equations, J. Difference Equ. Appl. 17 (2011), 445-456.

[4] F. M. ATici, P. W. Eloe, Linear systems of fractional nabla difference equations, Rocky Mountain J. Math. 41 (2011), 353-370.

[5] F. M. Atici, S. ŞEnGÜL, Modeling with fractional difference equations, J. Math. Anal. Appl. 369 (2010), 1-9.

[6] F. M. Atici, M. Uyanik, Analysis of discrete fractional operators, Appl. Anal. Discrete Math. 9 (2015), 139-149.

[7] J. Baoguo, L. Erbe, C. S. Goodrich, A. Peterson, On the relation between delta and nabla fractional difference, Filomat, to appear.

[8] N. R. O. Bastos, D. Mozyrs Ka, D. F. M. Torres, Fractional derivatives and integrals on time scales via the inverse generalized Laplace transform, Int. J. Math. Comput. 11 (2011), 1-9.

[9] R. DAHAL, C. S. GoODRICH, A monotonicity result for discrete fractional difference operators, Arch. Math. (Basel) 102 (2014), 293-299.

[10] R. Dahal, C. S. Goodrich, Erratum to "R. Dahal, C. S. Goodrich, A monotonicity result for discrete fractional difference operators, Arch. Math. (Basel) 102 (2014), 293-299”, Arch. Math. (Basel) 104 (2015), 599-600.

[11] R. A. C. Ferreira, A discrete fractional Gronwall inequality, Proc. Amer. Math. Soc. 140 (2012), $1605-1612$.

[12] R. A. C. FERREIRA, Existence and uniqueness of solution to some discrete fractional boundary value problems of order less than one, J. Difference Equ. Appl. 19 (2013), 712-718.

[13] C. S. GoodRICH, Solutions to a discrete right-focal boundary value problem, Int. J. Difference Equ. 5 (2010), 195-216.

[14] C. S. Goodrich, On discrete sequential fractional boundary value problems, J. Math. Anal. Appl. 385 (2012), 111-124. 
[15] C. S. GoodRICH, On a first-order semipositone discrete fractional boundary value problem, Arch. Math. (Basel) 99 (2012), 509-518.

[16] C. S. Goodrich, A convexity result for fractional differences, Appl. Math. Lett. 35 (2014), 58-62.

[17] C. S. GOODRICH, Systems of discrete fractional boundary value problems with nonlinearities satisfying no growth conditions, J. Difference Equ. Appl. 21 (2015), 437-453.

[18] C. Goodrich, A. C. Peterson, Discrete Fractional Calculus, Springer (2015), doi: 10.1007/9783-319-25562-0.

[19] M. Holm, Sum and difference compositions and applications in discrete fractional calculus, Cubo $\mathbf{1 3}$ (2011), 153-184.

[20] B. Jia, L. Erbe, A. Peterson, Two monotonicity results for nabla and delta fractional differences, Arch. Math. (Basel) 104 (2015), 589-597.

[21] B. Jia, L. ERbe, A. Peterson, Convexity for nabla and delta fractional differences, J. Difference Equ. Appl. 21 (2015), 360-373.

[22] B. JiA, L. ERbe, A. Peterson, Some relations between the Caputo fractional difference operators and integer order differences, Electron. J. Differential Equations (2015), No. 163, pp. 1-7.

[23] G. Wu, D. Baleanu, Discrete fractional logistic map and its chaos, Nonlinear Dyn. 75 (2014), 283-287. 